\title{
Biodegradable Screws Containing Bone Morphogenetic Protein-2 in an Osteoporotic Rat Model
}

\author{
Eun-Sun Jin, M.D., ${ }^{1,2}$ Ji Yeon Kim, M.D., ${ }^{2}$ Bora Lee, M.D., ${ }^{3}$ JoongKee Min, M.D., ${ }^{2}$ Sang Ryong Jeon, M.D., ${ }^{2,4}$ \\ Kyoung Hyo Choi, M.D., ${ }^{2,5}$ Je Hoon Jeong, M.D., Ph.D. ${ }^{2,6}$ \\ Department of Internal Medicine,' Kyung Hee University School of Medicine, Seoul, Korea \\ Laboratory of Stem Cell Therapy, ${ }^{2}$ Asan Medical Center, University of Ulsan College of Medicine, Seoul, Korea \\ Department of Biostatistic Consulting, ${ }^{3}$ Soon Chun Hyang Medical Center, Bucheon, Korea \\ Department of Neurological Surgery, ${ }^{4}$ Asan Medical Center, University of Ulsan College of Medicine, Seoul, Korea \\ Department of Rehabilitation Medicine, ${ }^{5}$ Asan Medical Center, University of Ulsan College of Medicine, Seoul, Korea \\ Department of Neurosurgery, ${ }^{6}$ Soonchunhyang University Bucheon Hospital, Bucheon, Korea
}

Objective : The aim of this study was to evaluate the effect for biodegradable screws containing bone morphogenetic protein-2 (BMP-2) in an osteoporotic rat model.

Methods : Twenty-four female Wistar rat (250-300 g, 12 weeks of age) were randomized into four groups. Three groups underwent bilateral ovariectomy (OVX). Biodegradable screws with or without BMP-2 were inserted in the proximal tibia in two implantation groups. The extracted proximal metaphysis of the tibiae were scanned by exo-vivo micro-computed tomography. Evaluated parameters included bone mineral density (BMD), trabecular bone volume (BV/TV), trabecular number, trabecular thickness, and trabecular separation (Tb.Sp). The tibia samples were pathologically evaluated by staining with by Hematoxylin and Eosin, and trichrome.

Results : Trabecular formation near screw insertion site was evident only in rats receiving BMP-2 screws. BMD and BV/TV significantly differed between controls and the OVX and OVX with screw groups. However, there were no significant differences between control and OVX with screw BMP groups. Tb.Sp significantly differed between control and OVX and OVX with screw groups $(p<0.05)$, and between the OVX and OVX with screw BMP group $(p<0.05)$, with no statistically significant difference between control and OVX with screw BMP groups. Over the 12 weeks after surgery, bone lamellae in direct contact with the screw developed more extensive and thicker trabecular bone around the implant in the OVX with screw BMP group compared to the OVX with screw group.

Conclusion : Biodegradable screws containing BMP-2 improve nearby bone conditions and enhance ostoeintegration between the implant and the osteoporotic bone.

Key Words : Osteoporosis · Models, Animal · Screw, Animal · Bone morphogenetic protein 2.

- Received : November 30, 2017 •Revised : January 11, 2018 •Accepted : February 9, 2018

- Address for reprints : Je Hoon Jeong, M.D., Ph.D.

Department of Neurosurgery, Soonchunhyang University Bucheon Hospital, 170 Jomaru-ro, Bucheon 14584, Korea

Tel : +82-32-621-6709, Fax : +82-32-621-5016, E-mail : neuri71@gmail.com

This is an Open Access article distributed under the terms of the Creative Commons Attribution Non-Commercial License (http://creativecommons.org/licenses/by-nc/4.0) which permits unrestricted non-commercial use, distribution, and reproduction in any medium, provided the original work is properly cited. 


\section{INTRODUCTION}

Postmenopausal osteoporosis is a disease characterized by low bone mass and depleted bony microarchitecture because of increasing bone turnover induced by estrogen deficiency ${ }^{15,33)}$. Implant insertion is very important in the surgical treatment of bone fracture for patients with osteoporosis. The reduction in bone mass lessens the capacity of bone to support an orthopedic implant ${ }^{6,15)}$. Consequences can include screw loosening or pull out, and delayed deformity of adjacent structures.

Various attempts to reinforce the integration of implants with bone and screws have been reported ${ }^{7,11,15,24,25)}$. In this study, the tactics that was explored was the use of biodegradable screws containing bone morphogenetic protein-2 (BMP-2) in an osteoporotic rat model.

\section{MATERIALS AND METHODS}

\section{Animals}

This study was approved by the Institutional Animal Care and Use Committee of Asan Institute for Life Sciences, Seoul, Republic of Korea. Twenty-four female Wistar rats (250-300 g, 12 weeks of age) were divided into four groups : sham (control, sham-operated) group, ovariectomy (OVX)-induced osteoporosis group, OVX and biodegradable screw (OVX with screw) group, and OVX and BMP-2 containing biodegradable screw (OVX with screw BMP) group.

\section{Ovariectomy}

Rats were anesthetized with 5\% isoflurane. Each received a $4 \mathrm{~cm}$ bilateral dorsal skin incision. Dissection was done to the retroperitoneal space to reveal the ovary surrounded by a whitish fat tissue. After ligation of the blood vessels, the connection between the fallopian tube and the uterine horn was severed. Each rat received an intra-muscular injection of antibiotic (cefazole $10 \mathrm{mg} / \mathrm{kg}$, ketoprofen $5 \mathrm{mg} / \mathrm{kg}$ ) daily for 3 days postoperatively. Body weight was measured once each 2 week for 16 weeks. Body weight was measured once per week for 16 weeks during the experimental period to ensure that animal models remain stable.

\section{Screw modification}

The Inion CPS ${ }^{\circledR}$ L-lactide biodegradable screws (Inion, Tampere, Finland) were $2 \mathrm{~mm}$ in outer diameter and $5 \mathrm{~mm}$ in length.
Two $0.5 \mathrm{~mm}$ sized perpendicularly crossed holes were made (Fig. 1A). An absorbable collagen sponge (Collatape ${ }^{\circledR}$; Zimmer Dental, Carlsbad, CA, USA) was used as a carrier for retention and delivery of recombinant human BMP-2 (rhBMP-2; SigmaAldrich, St. Louis, MO, USA) at the implantation site (Fig. 1B and C) A piece of Collatape ${ }^{\circledR}(1 \times 5 \mathrm{~mm})$ was inserted into the hole. Sterile water solution alone or containing 0.5 mg BMP-2 was applied to a collagen sponge, resulting in a final implant BMP-2 concentration of $4 \mathrm{ug} / \mathrm{mL}$ of BMP-2.

\section{Implantation}

Implantation in the ovariectomized rats was delayed by 4 weeks following the procedure to allow osteoporotic changes to occur. On the first day of week 5, each rate received a 5-6 mm longitudinal skin incision along the tibia. After soft tissue dissection, a hole was drilled using a 1.4-mm electronic drill $5 \mathrm{~mm}$ distal from the proximal tibial growth plate. A screw was inserted in the hole (Fig. 2A). The soft tissue was sutured and each rat received an intra-muscular injection of aforementioned antibiotic for the next 3 days.

\section{Micro-computed tomography (micro-CT) evaluation}

Sixteen weeks after OVX, tibiae were extracted and surface soft tissues were removed prior to structural and functional

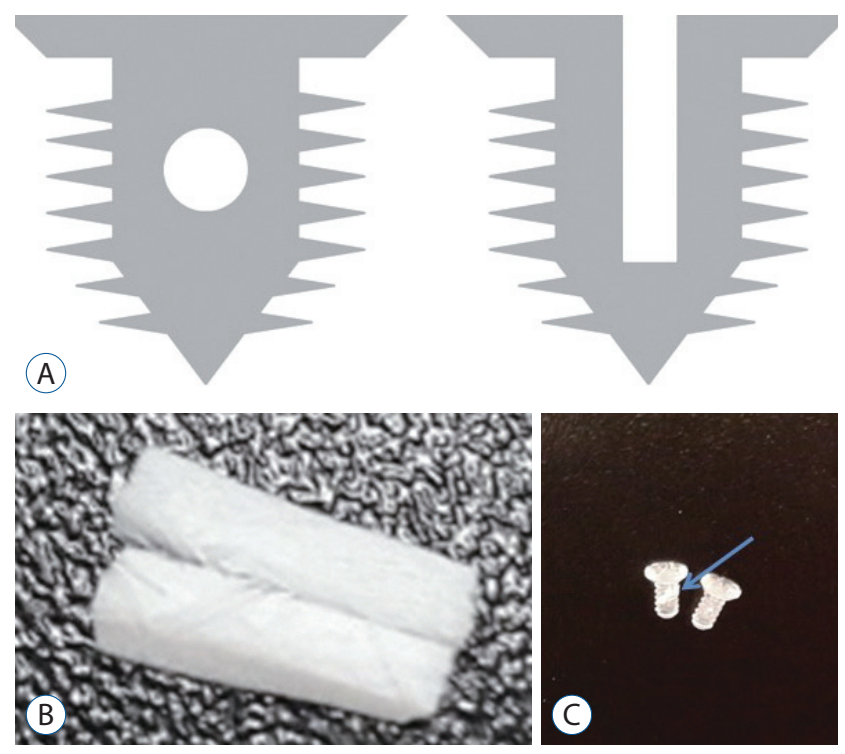

Fig. 1. Details of screw modification. A : Two $0.5 \mathrm{~mm}$ perpendicuallry crossed holes were made. $B$ and $C: A 1 \times 5 \mathrm{~mm}$ absorbable collagen sponge (Collata$\mathrm{pe}^{\circledast}$; Zimmer Dental, Carlsbad, CA, USA) was inserted in the hole (arrow) as a carrier for the delivery of bone morphogenetic protein-2. 


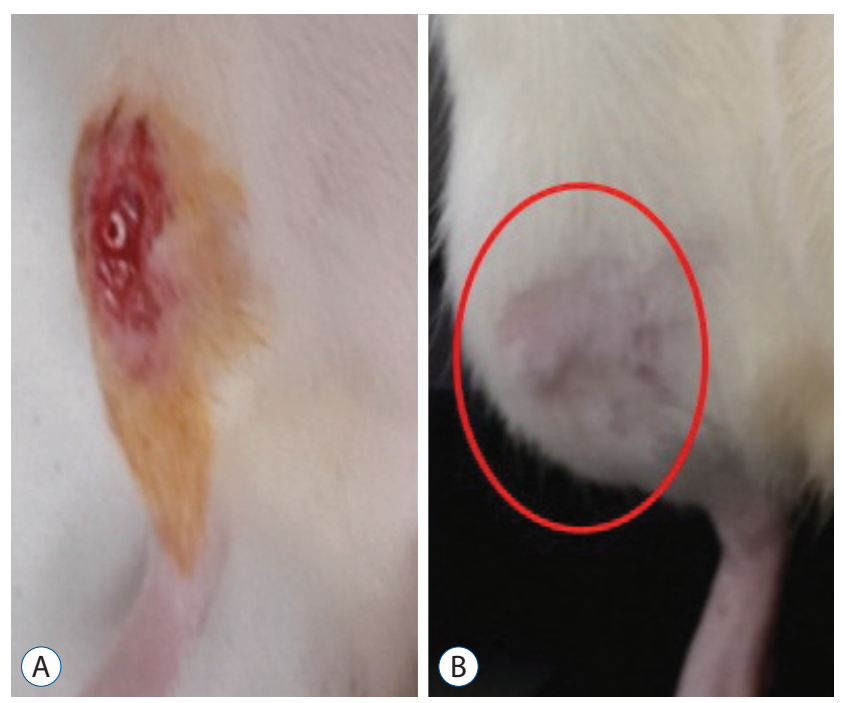

Fig. 2. Details of screw implantation. A : After dissection of soft tissue, a hole was made using a 1.4- $\mathrm{mm}$ rotary drill $5 \mathrm{~mm}$ distal from the proximal growth plate, and a screw was inserted. B : There is no wound site infection, dehiscence or swelling of the soft tissue (circle).

measurements. The samples were scanned by high-resolution micro-CT using a Skyscan 1176 micro-CT apparatus (Skyscan NV, Kontich, Belgium). The volume of interest (VOI) was defined distal from the growth plate and extending towards the diaphysis for $7.1 \mathrm{~mm}$ at a distance of $1.0 \mathrm{~mm}$ (340 slices). In each transverse slice of the VOI, the region of interest was defined as the entire trabeculae around the implants including the near implant area. The acquisition settings were as follows : X-ray source voltage $49 \mathrm{kV}$ and current $200 \mu \mathrm{A}$. A $0.5 \mathrm{~mm}$-thick aluminum filter was used to reduce bean hardening artifacts. The pixel size was $8.7 \mathrm{~mm}$ in a $4000 \times 2096$ charge-coupled device (CCD) detector array. The exposure time was 670 ms using a stepwise and rotation of $0.4^{\circ}$ with complete rotation achieved. Image reconstruction and analysis were performed using Skyscan software. The parameters evaluated included trabecular bone volume (BV/TV; \%), trabecular number (Tb.N; $/ \mathrm{mm}$ ), trabecular separation (Tb.Sp; mm), trabecular thickness (Tb.Th; $\mathrm{mm}$ ) and bone mineral density (BMD; $\left.\mathrm{g} / \mathrm{cm}^{2}\right)$.

\section{Hematoxylin and Eosin (H\&E) staining}

After decalcification, the tibia samples were fixed in paraffin and cut longitudinally at a thickness of $3 \mu \mathrm{m}$ using a model RM2255 microtome (Leica Microsystems, Wetzlar, Germany). Each section was mounted on a slide and put in a $55^{\circ} \mathrm{C}$. The sections were de-paraffinized in three 5-minute changes of xylene and dehydrated in three 5-minute changes in sequentially in- creasing alcohol solutions $(70 \%, 80 \%, 90 \%$, and $100 \%)$. The slides were rinsed in flowing distilled water for 1 minute and immersed in Harris Hematoxylin-I (YD-Diagnostics.com, Yongin, Korea) for 5 minutes, followed by a 1-minute wash in flowing tap water. The sections were then dehydrated from $70 \%$ to $100 \%$ ethanol and stained with Eosin Y (Sigma-Aldrich) for 30 seconds. Residual stain was washed off in a flow of distilled water. Each section was mounted (Shandon Synthetic Mountant; Thermo Fisher Scientific, Waltham, MA, USA).

\section{Masson's trichrome staining}

After staining of nuclei using an iron-hematoxylin kit (VWR International $\mathrm{GmbH}$, Lerzenstrasse, Switzerland), samples were stained for 15 minutes using azophloxin. Samples were washed using 1\% acetic acid, placed it in acid Orange G (Sigma, St. Louis, MO, USA) solution until collagen decolorixed and rinse with $1 \%$ acetic acid repeatedly for 30 seconds each time. Samples were stained with Light Green (Sigma) for 5 minutes and rinsed in $1 \%$ acetic acid repeatedly for 5 minutes.

\section{Statistical analyses}

Data were represented as means \pm standard deviations. According to the result of normality test, two-way repeated measures analysis of variance was applied to compare the change of body weight per group. Post hoc comparison was conducted at each time point which showed statisticially significant difference with student's t-test and Bonferroni's correction. Comparing micro-CT measurements among groups, Kruskal-Wallis test was performed and post hoc comparison was done at each value with Mann-Whitney $\mathrm{U}$ test and Bonferroni's correction. A two-tailed $p<0.05$ was considered as statistically significant. All statistical analyses were performed using R (version 3.3.2; The R Foundation for Statistical Computing, Vienna, Austria).

\section{RESULTS}

All animals tolerated the procedure well and rats could walk on all four legs immediately postoperatively. Most of the rats in the OVX with screw BMP group experienced mild swelling at implantation sited for 3 to 7 days after implantation. There were no cases of infection or wound dehiscence, and no complications related to screw implantation during the experimental period (Fig. 2B). No animals died or had to be euthanized. 


\section{In vitro observations}

An initial burst (about 50\%) in BMP-2 release occurred within the first 7 hours after contact with phosphate-buffered saline, followed by a steady release of 0.1 to $0.4 \%$ per day thereafter (Fig. 3).

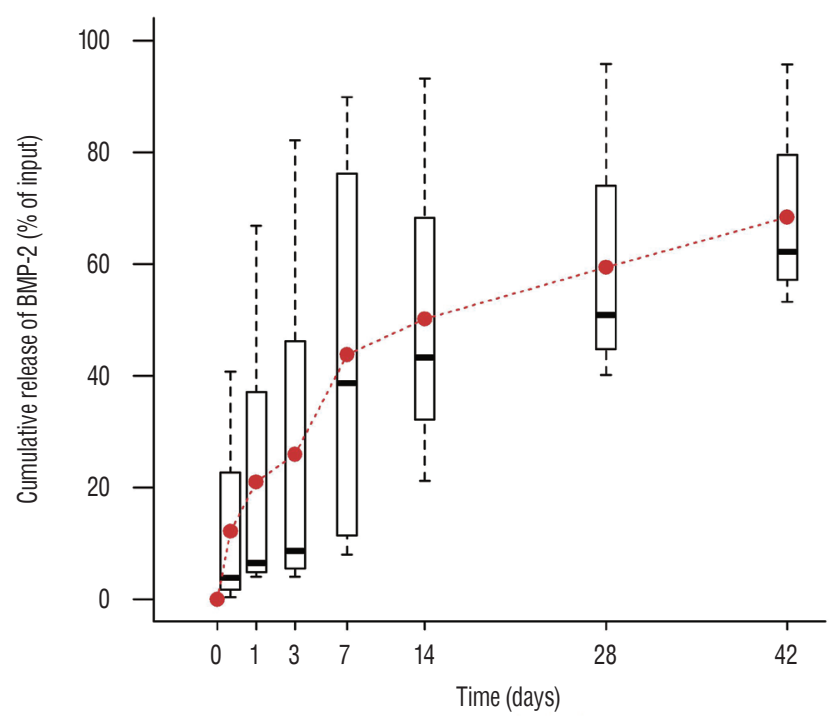

Fig. 3. In vitro BMP-2 release. An initial peak of release was evident in the first 2 hours after contact with fluids, with a steady release of 0.1 to $0.4 \%$ per day in the following days. BMP-2 : bone morphogenetic protein- 2 .

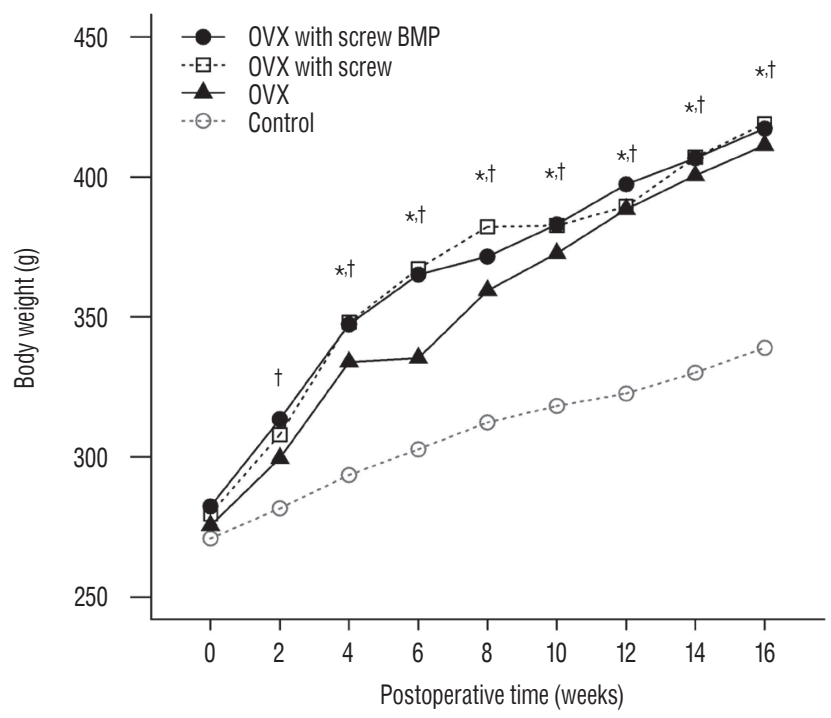

Fig. 4. Changes of the body weight of rats. The mean body weight increased over the 12 weeks. Body weight in the OVX, OVX with screw and OVX with screw BMP groups was higher than the control group $(p<0.05)$. *There exists the significant difference between control and OVX with screw. ${ }^{\dagger}$ There exists the significant difference between control and OVX with screw BMP. OVX : ovariectomy, BMP : bone morphogenetic protein.

\section{Body weight}

There was no significant difference in the body weight between the four groups at baseline. Body weight in the rats that underwent ovariectomy (OVX, OVX with screw, and OVX with screw BMP group) were higher than the control group. Total body weight differed significantly between the control and the other groups $(p<0.05)$ (Fig. 4).

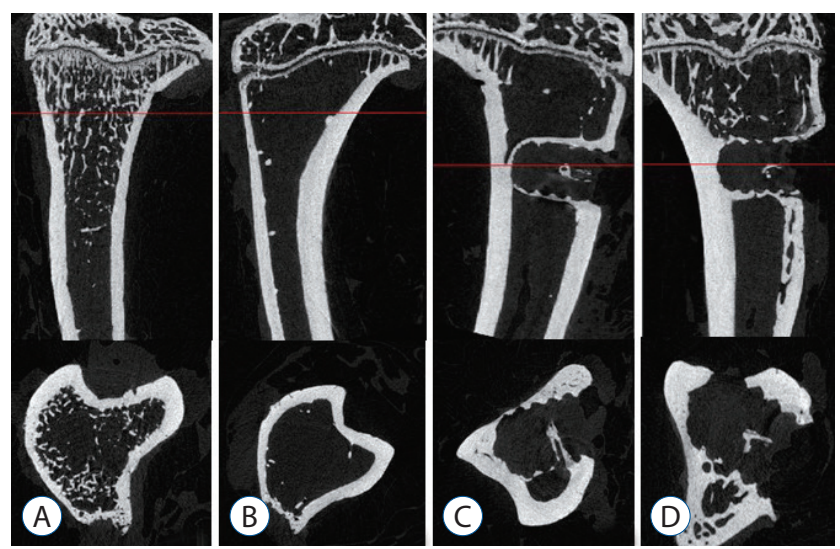

Fig. 5. Representative micro-computed tomography images (upper : sagittal, lower : axial), photographs showing osteoporotic changes of bone in the OVX, OVX with screw and OVX with screw BMP groups. Definite trabecular formation was evident near the screw insertion site only in the OVX with screw BMP group. A : Sham (control) group, B : OVX-induced osteoporosis group, $C$ : OVX and biodegradable screw (OVX with screw) group, D: OVX and BMP-2 containing biodegradable screw (OVX with screw BMP) group. OVX: ovariectomy, BMP : bone morphogenetic protein.

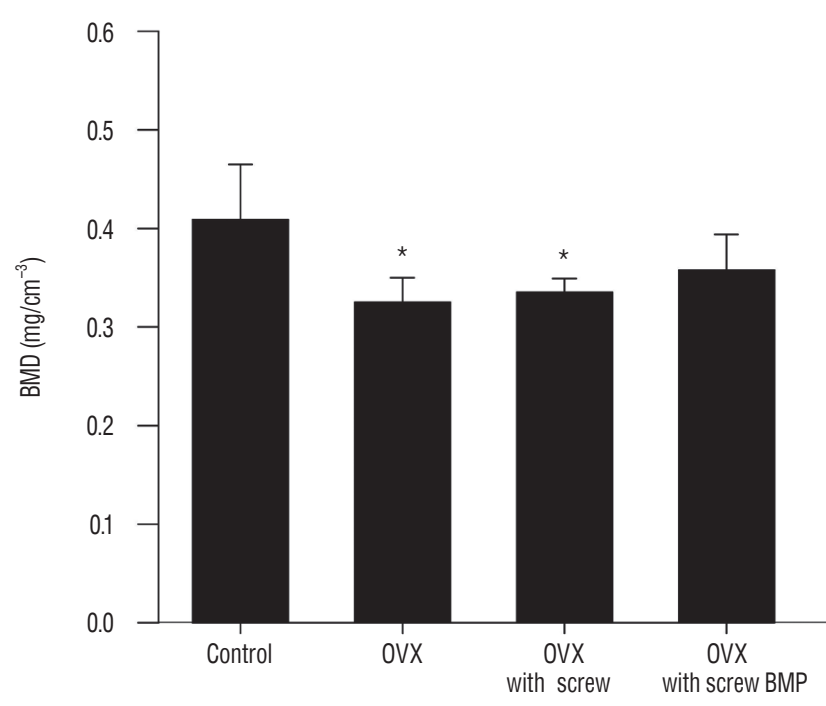

Fig. 6. Changes of BMD. BMD of the control group was the highest, with a significant difference between the control and OVX and OVX with screw groups $(p<0.05)$. * $p<0.05$ compared with control. OVX : ovariectomy, BMP: bone morphogenetic protein. 


\section{Micro-CT}

Representative micro-CT images shown in Fig. 5 illustrate the osteoporotic changes of bone in OVX, OVX with screw, and OVX with screw BMP groups after ovariectomy. New trabecular formation near the site of screw insertion was evident only in the OVX with screw BMP group (Fig. 5). BMD (Fig. 6) and BV/TV (Fig. 7A) of the control group were highest of all four groups, with a significant difference between the control and OVX and OVX with screw groups $(p<0.05)$, No significant difference was evident between the control and OVX with screw
BMP group ( $p>0.05)$. Tb.N displayed the same trend (Fig. 7B). However, there was a significant difference between the control group and the other groups $(p<0.05)$. Tb.Sp of the control group was lowest among all the groups (Fig. 7D). There was a significant difference between the control and OVX and OVX with screw groups $(p<0.05)$, but there was no statistically significant difference between the control and OVX with screw BMP groups $(p>0.05)$. A significant difference was also apparent in the OVX and OVX with screw BMP groups $(p<0.05)$ (Fig. 7).
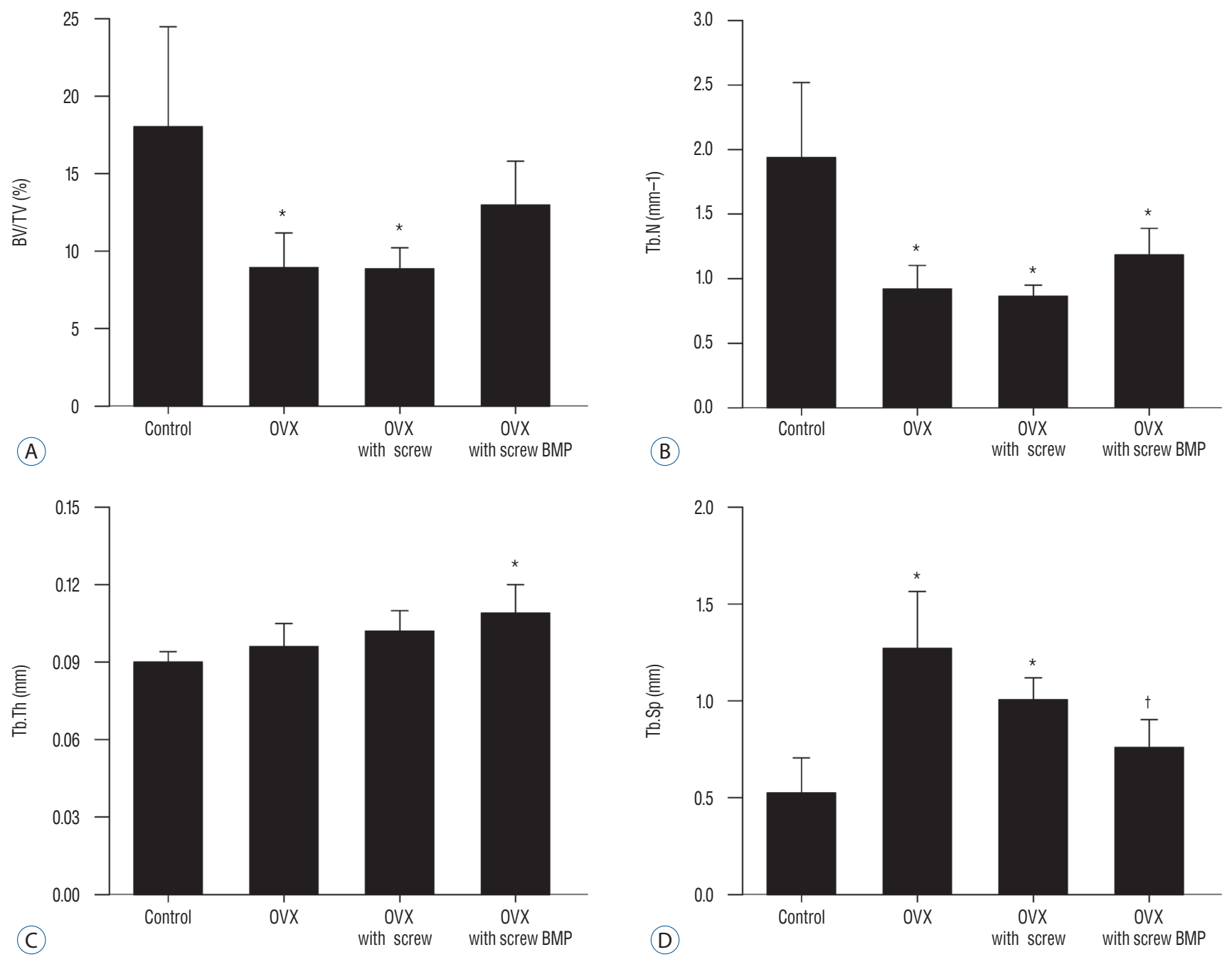

Fig. 7. Summary of micro-computed tomography findings. A : BV/TV of the control group was highest, with a significant difference between the control and OVX and OVX with screw groups $(p<0.05)$. B : Significant difference in Tb.N was evident between control and the other groups $(p<0.05)$. C: Tb.Th of the OVX with screw BMP group was highest, with a significant difference between the control and OVX with screw BMP groups $(p<0.05)$. D: Tb.Sp of the control group was lowest. There was a significant difference between the control and OVX and OVX with screw groups $(p<0.05)$, but there was no statistically significant difference between the control and OVX with screw BMP groups. A significant difference was evident between the OVX and OVX with screw BMP groups ( $p<0.05)$. * $p<0.05$ compared with control. ${ }^{\dagger} p<0.05$ compared with OVX. BV/TV : bone volume/trabecular volume, OVX : ovariectomy, Tb.N : trabecular number, Tb.Th : trabecular thickness, $\mathrm{BMP}$ : bone morphogenetic protein, Tb.Sp: trabecular separation. 


\section{Histology}

Over the 16 weeks after ovariectomy, histologic findings of $\mathrm{H} \& \mathrm{E}$ and Masson's trichrome staining revealed no inflammation or negative host response in any sample. There was less granulation tissue and more organized bone formation (Figs. 8 and 9). Histology of tibia revealed decreasing bony trabeculae and increased proportion of adipose tissue in the bone marrow in the OVX, OVX with screw and OVX with screw BMP groups compared with the control group (Figs. 8 and 9). The OVX with screw BMP group showed better findings than the OVX and OVX with screw groups, although not to the level of the control group. Thicker trabecular bone was observed in the bone lamellae of the direct contact area with the screw in the OVX with screw BMP group better than the OVX with screw group. In comparison with the OVX with screw group, mineralized collagen around the screw contact area appeared greatest and most organized in the OVX with screw BMP group (Fig. 9).

\section{DISCUSSION}

Orthopedic implant insertion is an accepted treatment of skeletal disease ${ }^{1,16)}$. Various systemic conditions including postmenopausal osteoporosis affect host bone structure and can diminish the long-term success of implants ${ }^{9,16,18,19)}$. The increased bone turnover that occurs with estrogen deficiency causes postmenopausal osteoporosis, which is low bone mass and worsening of bone microarchitecture ${ }^{15,33)}$. This can decrease in support of an orthopedic implant ${ }^{6,15)}$. Without appropriate treatment for osteoporosis, orthopedic implant fixation can be delayed and the procedure can ultimately fail ${ }^{15,17,30)}$. Bone grafting is a typical conventional procedure to enhance the osteointegration between host bone and orthopedic implant. Complications include infection, donor-site morbidity and prolonged rehabilitation $^{2,12)}$.

BMP first identified by Urist $^{27)}$ as a signal transduction ma-
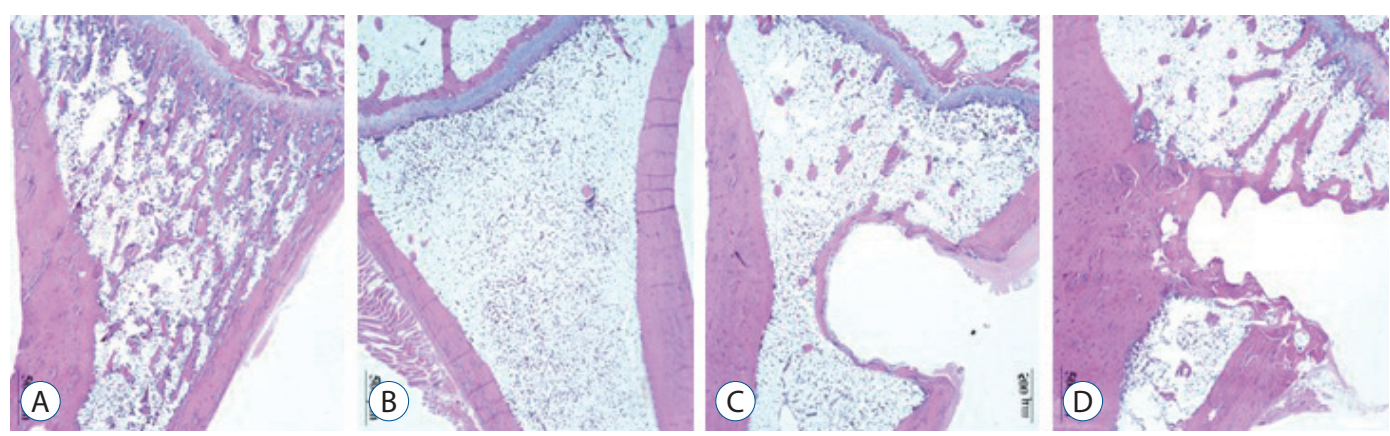

Fig. 8. Representative photographs of the H\&E staining of the tibia. Disappearance of the bony trabeculae and increased of adipose tissue proportion at the bone marrow in the OVX, OVX with screw and OVX with screw BMP groups were apparent compared with the control group. Although not to the level of the control group, this condition was better than in the OVX with screw BMP group. In the bone lamellae in direct contact with the screw, there were more extensive and thickened trabecular bone around the implant in the OVX with screw BMP group than the OVX with screw group (Magnification, $\times 20$ ). A : Sham (control) group, B : OVX-induced osteoporosis group, C:OVX and biodegradable screw (OVX with screw) group, D: OVX and BMP-2 containing biodegradable screw (OVX with screw BMP) group. OVX: ovariectomy, BMP: bone morphogenetic protein.
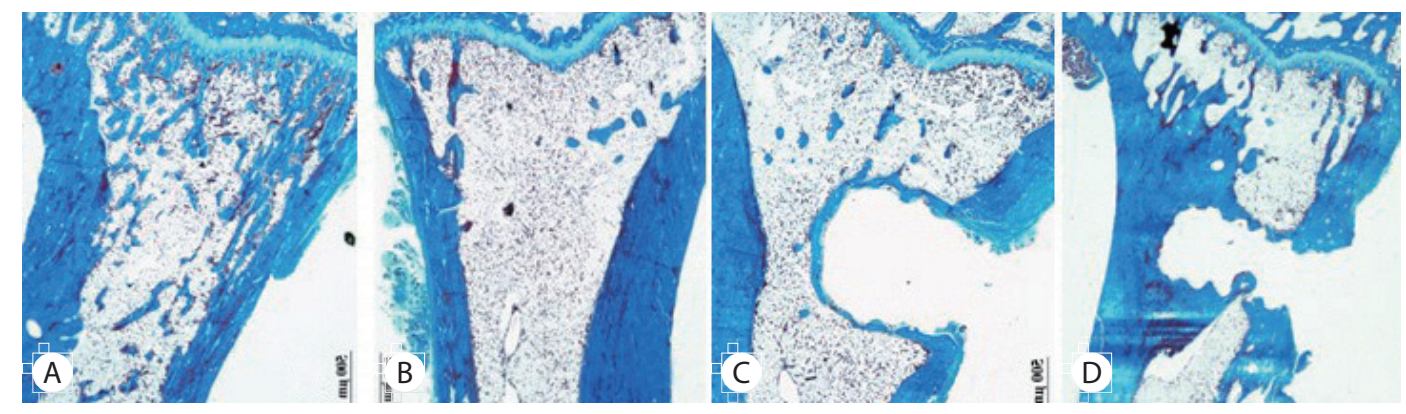

Fig. 9. Representative photographs of Masson's trichrome staining. Mineralized collagen around the contact area of the screw appeared most prominent and most organized in the OVX with screw BMP group (magnification, $\times 20$ ). A : Sham (control) group, B: OVX-induced osteoporosis group, C: OVX and biodegradable screw (OVX with screw) group, D: OVX and BMP-2 containing biodegradable screw (OVX with screw BMP) group. OVX: ovariectomy, BMP: bone morphogenetic protein. 
terial belonging to transforming growth factor-beta is capable of osteoinduction-inducing osteogenesis from mesodermal cells $^{2,32}$. BMP-2 helps promote cell growth and differentiation $^{2,14,31)}$, and acts directly on the induction and differentiation of osteoblasts, which participate in fracture healing and the regeneration of damaged bone tissue ${ }^{2,14)}$. The advent of rhBMP manufacture has allowed the mass production of BMP. The safety and excellence of rhBMP-2 has been demonstrated ${ }^{14,32}$. However, problems associated with BMP that remain include immune response, high cost, stimulation of unwanted ectopic bone formation and tumor formation ${ }^{4,14}$. Furthermore, BMP is typically supplied as a liquid, which complicated its use during surgery. It is difficult to induce ideal bone formation because BMP diffuses and is absorbed at a very high rate at the injection site ${ }^{14,29)}$. Establishing a suitable scaffold system to deliver the appropriate dose of BMP is an important clinical goal.

The ideal delivery system of BMP in the orthopedic implant provides sufficient space for bone growth, mechanical strength as a structural support, excellent biocompatibility, allows the constant release of an appropriate quantity of the material ${ }^{14)}$, maximizes bone formation, has a porous structure that induces cell attachment and cell infiltration ${ }^{28)}$ and is safely degraded and reabsorbed ${ }^{10,13,14,29)}$. A suitable biocompatible system for the delivery of BMP to maximize bone regeneration remains a challenge ${ }^{28}$. In previous study described the use of a collagen sponge to release rhBMP-2 as a means of rapidly inducing osteogenesis by osteoprogenitor cells ${ }^{3,8)}$. Because of this fast release, rhBMP rapidly diffuses to the fracture site before reaching a critical density for the new infiltration of cells ${ }^{8}$. Another study reported that prior to new bone formation in the metaphyseal defects, fast release of rhBMP produces osteoclast-mediated resorption $^{3,8,26)}$. While the limitations of the collagen sponge have been well documented, there is a lack of evidence demonstrating superior release kinetics of other delivery systems. Hence, we chose the collagen sponge as the delivery system in this study. Collatape can absorb BMP quickly, has excellent biocompatibility and a rapid biodegradable rate. However, Collatape lacks sufficient mechanical strength and early bone resorption of BMP can occur; both can induce bone weakness ${ }^{28)}$. We used a modified biodegradable screw as the structural support. The modified screw that featured a central canal that housed the BMP-2 soaked collatape proved to be a good system for the delivery of BMP for the induction of osteogenesis. Therefore, micro-CT scans revealed trabecular formation near the screw insertion site only in the OVX with screw BMP group (Fig. 5). Measurements of BMD and BV/TV indicated the efficacy of the screw system.

The dose of BMP used in bone healing treatment is a crucial safety concern. The dose must be high enough to control the biological regulation of osteoblastic and osteoclastic factors ${ }^{22,28)}$ and involves an amount of progenitor cells that is sufficient to form new bone ${ }^{23,28)}$. However, very high BMP dose leads to excessive edema and inflammation, as well as formation of cystic change of the bone, or ectopic bone formation ${ }^{15,20,24}$. The appropriate dose of BMP remains contentious, with different effective concentrations claimed based on different animal models and study environments ${ }^{15}$. A bone defect rat model reported that the minimum amount to achieve femoral defect filling using a non-glycosylated BMP-2 with a fibrin matrix was $5 \mu \mathrm{g} / \mathrm{mL}^{21,28)}$. Another study reported doses less than $5 \mu \mathrm{g}$ of non-glycosylated BMP-2 was not sufficient to achieve vertical bone regeneration using a collagen/hydroxyapartite/synthetic polyethylene glycol-hydrogel scaffold/titanium implant combination in a rat mandible model ${ }^{28}$. It should be noted that the $5 \mu \mathrm{g}$ dose was the lowest and most effective dose in the latter study. Another study of rat calvarial defect model reported that $5 \mu \mathrm{g}$ of BMP was too low, although more than $90 \%$ of rat calvarial defects were regenerated during 12 weeks, the new bone was only $65 \%$ of the density of the native bone under this density of $\mathrm{BMP}^{5,28)}$. In the present study, the model was not bone defect model. We used $4 \mu \mathrm{g} / \mathrm{mL}$, which is relatively lower dose to the lower limit of the osteogenic threshold. In this study, there was thicker trabecular bone was observed in the bone lamellae of direct contact area with the screw in the OVX with screw BMP group better than OVX with screw group, although not to the level of the control group. In comparison with the OVX with screw group, mineralized collagen around the contact area of the screw appears greatest and most organized in the OVX with screw BMP group (Fig. 9). Furthermore, because of the safety concern, a low dose of BMP is better, in terms of minimizing complications like severe swelling and osteoclastic bone changes.

In this study, most of the rats of the OVX with screw BMP group exhibited mild swelling for 3 to 7 days after implantation. However, none had issues of infection or wound dehiscence, and there were no complications concerned with implantation of the screws in experimental period (Fig. 2). Histologic findings of H\&E and Masson's trichrome staining showed no inflammation or negative host response in any sample. There 
was also less granulation tissue and more organized bone formation (Figs. 8 and 9).

\section{CONCLUSION}

Biodegradable screws containing BMP-2 can improve nearby bone conditions and enhance ostoeintegration between the implant and the osteoporotic bone.

\section{CONFLICTS OF INTEREST}

No potential conflict of interest relevant to this article was reported.

\section{INFORMED CONSENT}

Informed consent was obtained from all individual participants included in this study.

\section{- Acknowledgements}

This work was supported by the National Research Foundation of Korea (NRF) grant funded by the Korea government (MSIT) (grant number : NRF-2017R1A2B1005327), and this work was supported by the Soonchunhyang University Research Fund.

\section{References}

1. Astrand P, Ahlqvist J, Gunne J, Nilson H : Implant treatment of patients with edentulous jaws: a 20-year follow-up. Clin Implant Dent Relat Res $10: 207-217,2008$

2. Brown KV, Li B, Guda T, Perrien DS, Guelcher SA, Wenke JC : Improving bone formation in a rat femur segmental defect by controlling bone morphogenetic protein-2 release. Tissue Eng Part A 17 : 1735-1746, 2011

3. Cho TJ, Gerstenfeld LC, Einhorn TA : Differential temporal expression of members of the transforming growth factor beta superfamily during murine fracture healing. J Bone Miner Res 17 : 513-520, 2002

4. Choi H, Park NJ, Jamiyandorj O, Choi KH, Hong MH, Oh S. Improvement of osteogenic potential of biphasic calcium phosphate bone substitute coated with two concentrations of expressed recombinant human bone morphogenetic protein 2. J Periodontal Implant Sci 42 : 119-126, 2012
5. Cowan CM, Aghaloo T, Chou YF, Walder B, Zhang X, Soo C, et al. : MiCroCT evaluation of three-dimensional mineralization in response to BMP-2 doses in vitro and in critical sized rat calvarial defects. Tissue Eng $13:$ 501-512, 2007

6. Duarte PM, César Neto JB, Gonçalves PF, Sallum EA, Nociti jF : Estrogen deficiency affects bone healing around titanium implants: a histometric study in rats. Implant Dent $12:$ 340-346, 2003

7. Gao Y, Zou S, Liu X, Bao C, Hu J : The effect of surface immobilized bisphosphonates on the fixation of hydroxyapatite-coated titanium implants in ovariectomized rats. Biomaterials 30 : 1790-1796, 2009

8. Hämmerle CH, Olah AJ, Schmid J, Flückiger L, Gogolewski S, Winkler JR, et al. : The biological effect of natural bone mineral on bone neoformation on the rabbit skull. Clin Oral Implants Res 8 : 198-207, 1997

9. Herrmann I, Lekholm U, Holm S, Kultje C : Evaluation of patient and implant characteristics as potential prognostic factors for oral implant failures. Int J Oral Maxillofac Implants 20 : 220-230, 2005

10. Hyun SJ, Han DK, Choi SH, Chai JK, Cho KS, Kim CK, et al. : Effect of recombinant human bone morphogenetic protein-2, -4 , and -7 on bone formation in rat calvarial defects. J Periodontol 76 : 1667-1674, 2005

11. Jakobsen T, Baas J, Kold S, Bechtold JE, Elmengaard B, Søballe K : Local bisphosphonate treatment increases fixation of hydroxyapatite-coated implants inserted with bone compaction. J Orthop Res 27 : 189-194, 2009

12. Jung JH, Yun JH, Um YJ, Jung UW, Kim CS, Choi SH, et al. : Bone formation of Escherichia coli expressed rhBMP-2 on absorbable collagen block in rat calvarial defects. Oral Surg Oral Med Oral Pathol Oral Radiol Endod 111 : 298-305, 2011

13. Jung UW, Choi SY, Pang EK, Kim CS, Choi SH, Cho KS : The effect of varying the particle size of beta tricalcium phosphate carrier of recombinant human bone morphogenetic protein-4 on bone formation in rat calvarial defects. J Periodontol 77 : 765-772, 2006

14. Lee S, Choi H, Shim JS, Chung MK, Park YB : Comparative study of recombinant human bone morphogenetic protein-2 carriers in rat subcutaneous tissues: Pilot study. Tissue Eng Regen Med 12 : 138-146, 2015

15. Li Y, Feng G, Gao Y, Luo E, Liu X, Hu J : Strontium ranelate treatment enhances hydroxyapatite-coated titanium screws fixation in osteoporotic rats. J Orthop Res 28 : 578-582, 2010

16. Liang YQ, Qi MC, Xu J, Xu J, Liu HW, Dong W, et al. : Low-magnitude high-frequency loading, by whole-body vibration, accelerates early implant osseointegration in ovariectomized rats. Mol Med Rep 10 : 2835-2842, 2014

17. Lugero GG, de Falco Caparbo V, Guzzo ML, König B Jr, Jorgetti V : Histomorphometric evaluation of titanium implants in osteoporotic rabbits. Implant Dent 9 : 303-309, 2000

18. Mellado-Valero A, Ferrer-García JC, Calvo-Catalá J, Labaig-Rueda C : Implant treatment in patients with osteoporosis. Med Oral Patol Oral Cir Bucal 15 : e52-e57, 2010

19. Qi M, Hu J, Li J, Li J, Dong W, Feng X, et al. : Effect of zoledronate acid treatment on osseointegration and fixation of implants in autologous iliac bone grafts in ovariectomized rabbits. Bone 50 : 119-127, 2012

20. Qi MC, Zhou XQ, Hu J, Du ZJ, Yang JH, Liu M, et al. : Oestrogen replace- 
ment therapy promotes bone healing around dental implants in osteoporotic rats. Int J Oral Maxillofac Surg 33 : 279-285, 2004

21. Schmoekel $H$, Schense JC, Weber FE, Grätz KW, Gnägi D, Müller R, et al. : Bone healing in the rat and dog with nonglycosylated BMP-2 demonstrating low solubility in fibrin matrices. J Orthop Res 22 : 376-381, 2004

22. Seeherman H, Wozney J, Li R : Bone morphogenetic protein delivery systems. Spine (Phila Pa 1976) 27 : S16-S23, 2002

23. Seeherman $H$, Wozney JM : Delivery of bone morphogenetic proteins for orthopedic tissue regeneration. Cytokine Growth Factor Rev 16 : 329-345, 2005

24. Skoglund B, Holmertz J, Aspenberg P : Systemic and local ibandronate enhance screw fixation. J Orthop Res 22 : 1108-1113, 2004

25. Skripitz R, Böhling $S$, Rüther $W$, Aspenberg $P$ : Stimulation of implant fixation by parathyroid hormone (1-34)-A histomorphometric comparison of PMMA cement and stainless steel. J Orthop Res 23 : 1266-1270, 2005

26. Tägil $M$, Jeppsson $C$, Wang JS, Aspenberg $P$ : No augmentation of morselized and impacted bone graft by OP-1 in a weight-bearing model. Acta Orthop Scand 74 : 742-748, 2003

27. Urist MR : Bone morphogenetic protein: the molecularization of skeletal system development. J Bone Miner Res 12 : 343-346, 1997

28. Wen B, Karl M, Pendrys D, Shafer D, Freilich M, Kuhn L : An evaluation of BMP-2 delivery from scaffolds with miniaturized dental implants in a novel rat mandible model. J Biomed Mater Res B Appl Biomater 97 : 315-326, 2011

29. Wikesjö UM, Guglielmoni P, Promsudthi A, Cho KS, Trombelli L, Selvig $K A$, et al. : Periodontal repair in dogs: effect of rhBMP-2 concentration on regeneration of alveolar bone and periodontal attachment. J Clin Periodontol 26 : 392-400, 1999

30. Yamaguchi K, Masuhara K, Yamasaki S, Nakai T, Fuji T : Cyclic therapy with etidronate has a therapeutic effect against local osteoporosis after cementless total hip arthroplasty. Bone 33 : 144-149, 2003

31. Yamazaki Y, Oida S, Akimoto Y, Shioda S : Response of the mouse femoral muscle to an implant of a composite of bone morphogenetic protein and plaster of Paris. Clin Orthop Relat Res (234) : 240-249, 1988

32. Zheng LW, Wong MC, Rabie AB, Cheung LK : Evaluation of recombinant human bone morphogenetic protein-2 in mandibular distraction osteogenesis in rabbits: effect of dosage and number of doses on formation of bone. Br J Oral Maxillofac Surg 44 : 487-494, 2006

33. Consensus development conference: diagnosis, prophylaxis, and treatment of osteoporosis. Am J Med 94 : 646-650, 1993 